\title{
Synchronous Low Frequency Earthquakes and Implications for Deep San Andreas Fault Slip
}

(1)

\author{
Daniel T. Trugman ${ }^{1,2}$, Chunquan $\mathrm{Wu}^{1,3}$, Robert A. Guyer ${ }^{1,4}$, Paul A. \\ Johnson ${ }^{1}$.
}

${ }^{1}$ Geophysics Group (EES-17), Los Alamos National Laboratory, Los Alamos, NM 87545, USA

${ }^{2}$ Institute for Geophysics and Planetary Physics, Scripps Institution of Oceanography, San

Diego, California, USA

${ }^{3}$ Center for Earthquake Research and Information, University of Memphis, Memphis, Tennessee, USA

${ }^{4}$ Department of Physics, University of Nevada, Reno, Nevada, USA

*Corresponding author: D.T. Trugman, Institute for Geophysics and Planetary Physics, Scripps Institution of Oceanography, 9500 Gilman Drive, La Jolla, CA 92093-0225. Contact phone number: 505-412-7565. Contact email address: dtrugman@ ucsd.edu. 


\section{Abstract}

26 Low Frequency Earthquakes (LFEs) are slip events that occur repeatedly at

27 source locations within the lower crust. LFEs, and the associated seismic broadcast

28 known as tremor, have been observed in a diverse array of tectonic environments. Here

29 we develop a suite of statistical tools to conduct a systematic study of the spatial and

30 temporal correlations of the event occurrence patterns of the 88 LFE sources beneath the

31 greater Parkfield section of the San Andreas Fault. We first examine correlations in the

32 occurrence patterns on long time scales to show that the regions to the north and south of

33 Parkfield behave independently. We next use the cumulative event signatures of each

34 source to characterize the individual occurrence patterns on shorter time scales. Through

35 application of a statistical clustering algorithm, we demonstrate that individual LFE

36 sources form spatially coherent clusters that may represent localized elastic structures or

37 asperities on the deep fault interface. We conclude by examining the fine-scale features

38 of the event rates within the LFE occurrence patterns. Through quantitative comparison

39 to analogous laboratory shear experiments on granular, fault gouge-like materials, we

40 infer that that the distinctive features of LFE occurrence patterns reflect variations in the

41 in-situ stress and frictional conditions at the individual LFE source locations. These

42 observations provide a framework to understand the spatial and temporal diversity of

43 fault slip that occurs within the lower crust beneath Parkfield and that may influence

44 seismic hazard in the region.

46 Keywords: low frequency earthquakes; lower crustal deformation; acoustic emission,

$47 \quad$ Parkfield seismicity; earthquake clustering 


\section{1. Introduction}

49 The largest and most damaging earthquakes typically occur in the upper crust,

50 where the frictional regime is primarily brittle. Yet knowledge of the upper crust is not in

51 itself sufficient to characterize seismic hazard, as lower crustal slip beneath the

52 seismogenic zone can drive the seismicity in the upper crust (Tse and Rice 1986; Scholz

53 2002) and lead to the nucleation of large earthquakes (Nadeau and Guilhem 2009; Shelly

54 2009; Segall and Bradley 2012). Low Frequency Earthquakes (LFEs), which accompany

55 the slow slip events observed on the deep extension of fault interfaces in Cascadia

56 (Rogers and Dragert 2003; Bostock et al. 2012), Japan (Obara et al. 2004; Shelly et al.

57 2006), and Mexico (Rivet et al. 2011; Frank et al. 2013), have also been observed in the

58 Parkfield region of California (Nadeau and Dolenc 2005; Shelly et al. 2009), and present

59 the opportunity to illuminate the elusive elastic and slip behaviors of the lower crust

60 (Rubinstein, et al. 2010).

61 Low Frequency Earthquakes differ from classical earthquakes in their relative

62 deficiency in high frequency seismic radiation and in their location in the lower crust.

63 Shelly and Hardebeck (2010) identified 88 LFE sources in the Parkfield region of

64 California (Figure 1) by cross-correlating template waveforms with the continuous

65 seismic recordings at multiple High Resolution Seismic Network (HRSN) stations. The

66 seismic events associated with each LFE source occur repeatedly at average rates that

67 vary from source to source and range from 300 to 3000 events per year. LFE events at a

68 given source tend to occur in burst-like sequences of multiple events in rapid succession,

69 generating a nearly continuous seismic broadcast known as tectonic tremor (Shelly et al.

70 2007). Though the focus of this study is on the occurrence patterns of individual LFE 
71 events, we note that LFEs and tremor appear to be part of the same underlying

72 phenomena.

73 The $2004 \mathrm{M}_{\mathrm{w}} 6.0$ Parkfield earthquake transiently perturbed LFE activity in the

74 region (Shelly and Johnson 2011; Thomas et al. 2012; Wu et al. 2013). Consequently,

75 we restrict our analysis to the $6+$ year time interval $T$ from January $1^{\text {st }}, 2006$ to February

$761^{\text {st }}, 2012$. We use the comprehensive LFE catalog of Shelly and Hardebeck (2010), which

77 consists of 88 sets of time points corresponding to the times of LFE event occurrence for

78 each source. Each of the 88 LFE sources has a distinctive occurrence pattern, and we

79 proceed under the premise that these patterns reflect the source's individual slip behavior.

80 In this study, we examine correlations in LFE occurrence patterns over multiple

81 time and spatial scales. We first consider temporal correlations on timescales on the order

82 of days - correlations that reveal a clear decoupling between the LFE sources located to

83 the north of Parkfield from those to the south. We next characterize each individual LFE

84 source using its cumulative event record, and compute what we term a cumulative time

85 signature that quantifies the unique occurrence pattern of each source. By applying a

86 statistical clustering algorithm to the individual cumulative time signatures, we find that

87 the LFE sources at Parkfield naturally form spatially coherent patches, or clusters. These

88 clusters contain sources with quantitatively similar occurrence patterns, and may

89 represent coherent elastic structures or asperities on the deep fault interface. We conclude

90 with an analysis of the spectrum of event rates exhibited within the occurrence patterns of

91 each LFE source. Through quantitative comparison with laboratory shear experiments in

92 fault gouge-like materials, we demonstrate that the variability in these event rate spectra 
93 is likely caused by differences in the stress and frictional conditions experienced by each

94 LFE source.

\section{2. Methods and Results}

\section{2.1) Temporal correlations of periods of high LFE activity}

97 We begin our examination of the space-time correlations in LFE occurrence

98 patterns by tracking periods of time in which an individual source is particularly active.

99 To accomplish this, we break the observation interval (a total of 2222 days from January

$1001^{\text {st }}, 2006$ to February $\left.1^{\text {st }}, 2012\right)$ into discrete, 2-day time bins, and for each source, form

101 1111-component vectors (i.e., one component per time bin) containing the count of the

102 number of LFE events in each 2-day interval. To identify the periods of time in which

103 each LFE source is particularly active, we form corresponding binary, 1111-component

104 vectors in which the time bins with an event rate (events/time bin) greater than 3 standard

105 deviations above the mean rate are given a value of one. All other time bins are given a

106 value of zero. The values of mean and standard deviation in event rate are computed

107 individually for each source.

108 To facilitate comparison of the vectors $\left\{V_{n}\right\}$ for each of the $n$ sources, we remove

109 the mean component of each vector and normalize each vector to 1. In this way,

$110 \quad V_{i} \cdot V_{i}=1$ for any source $i$, and the inner product $V_{i} \cdot V_{i}$ provides a quantitative measure

111 of the temporal correlation of the periods of high LFE activity for any pair of sources $i$

112 and $j$. We compute these pairwise correlation coefficients $\rho_{i j}=V_{i} \cdot V_{j}$ for each of the

1133828 possible LFE source pairs $(i, j)$.

114 We display the results of this analysis in Figure 1c, in which the time bins

115 corresponding to high LFE event rates are denoted with a dot. Each LFE source is sorted 
116 by its distance, $Z$, from Parkfield $\left(35.9^{\circ} \mathrm{N}, 120.4^{\circ} \mathrm{W}\right)$ along the strike of the SAF. The

117 vertical streaking apparent in Figure 1c (e.g., among sources located between $Z=-20 \mathrm{~km}$

118 and $Z=-40 \mathrm{~km}$ along strike) is evidence of correlation of high event rates for nearby

119 sources. Strong spatiotemporal correlations are seen north and south of Parkfield ( $Z=0$

$120 \mathrm{~km}$ ), but these correlations do not appear to extend across the notable gap in LFE sources

121 at Parkfield.

122 We demonstrate this quantitatively by sorting the 3828 possible pairwise

123 correlation coefficients $\left(\rho_{i j}=V_{i} \cdot V_{j}\right)$ into three groups: (1) the 1128 pairs where both

124 sources reside to the south of Parkfield, (2) the 780 pairs where both sources reside to the

125 north of Parkfield, and (3) the remaining 1920 pairs with a single source from both the

126 north and south of Parkfield. The source pairs from group 1 (both south of Parkfield)

127 exhibit strong temporal correlation: $28.5 \%$ of all possible group 1 source pairs have

128 positive correlation coefficients that are statistically significant at the $99 \%$ confidence

129 level (obtain via Monte Carlo simulation). Similarly, 34.7\% of all possible group 2

130 source pairs (both north of Parkfield) exhibit statistically significant temporal correlation.

131 In contrast, only $0.4 \%$ of the group 3 source pairs (mixed north-south) exhibit this same

132 level of temporal correlation, indicating that the LFE sources north and south of Parkfield

133 are essentially decoupled. This result is robust with respect to the length of the

134 observation interval (i.e., time bin), effectively ruling out propagation of LFE activity

135 across the Parkfield gap at the slow velocities ( $10 \mathrm{~km} /$ day) observed in subduction

136 zones (Obara et al. 2004; Wech and Creager 2011).

137 Differences in LFE occurrence between the northern and southern sources have 138 been observed in several previous studies. Shelly and Hardebeck (2010) found that the 
139 seismic amplitudes of the southern sources tend to be larger than the amplitudes of the

140 northern sources. Guilhem and Nadeau (2012) found that the southern LFE sources

141 participate in quasi-periodic tremor episodes. Likewise, differences in sensitivity to static

142 stresses (Shelly and Johnson 2011), dynamic stresses (Shelly et al. 2011), and tidal

143 loading (Thomas et al. 2012) for the northern and southern sources have all been noted in

144 previous studies. Our results provide additional evidence that the northern and southern

145 segments of the deep San Andreas Fault behave independently.

\section{$146 \quad 2.2$ Clustering of Nearby LFE Sources}

147 We quantitatively characterize the distinctive features of the LFE event record of

148 each source by computing what we term the cumulative time signature of each LFE

149 source. There are three basic steps in forming the cumulative time signature, CTS(t), for

150 an individual LFE source (outlined pictorially in Figure S1 in the supplementary

151 material). We first compute $N(t)$, the fraction of LFE events occurring at or before time $t$.

152 We then subtract a quadratic polynomial fit from $N(t)$, resulting in $d N(t)$. For this step, we

153 use a quadratic (rather than linear) fit for the long-term trend because the event rate

154 decreases as time advances; this slow decrease in event rate may be related to the long-

155 term recovery process from the effects of the $2004 \mathrm{M}_{\mathrm{w}}$ 6.0 Parkfield earthquake (Shelly

156 and Johnson 2011; Wu et al. 2013). The final cumulative time signature, CTS(t), is

157 obtained by interpolating $d N(t)$ to 8912 time points that are uniformly distributed over the

158 observation interval $T$, removing the low-frequency Fourier components of $d N(t)$ (those

159 with periods greater than 100 days), and normalizing the resulting vector to 1 .

160 The cumulative time signature provides a fingerprint that quantitatively represents

161 the occurrence patterns of each LFE source over 6+ years from which the slowly varying, 
162 long-term trends have been removed (Figure S2 in the supplementary material). Further,

163 the $C T S(t)$ allow for a quantitative comparison of the occurrence patterns among the

164 sources. To measure the similarity between the occurrence patterns of two distinct LFE

165 sources, $m$ and $n$, we use the maximum value of the cross-correlation between the $C T S(t)$ :

$166 S_{m, n}=\max _{r}\left(\operatorname{cts} S_{m}(t) \cdot \operatorname{crs} S_{n}(t+\tau)\right]$

167 Pairs of sources with similar occurrence patterns have a higher $S$ than those with less

168 similar occurrence patterns, with $S$ approaching one in the limit that the patterns are

169 identical (i.e., $S_{n n}=1$ for all $n$ ).

170 Previous studies have hinted at the possibility that certain groups of nearby

171 sources tend to exhibit qualitatively similar occurrence patterns (Shelly 2010b; Guilhem

172 and Nadeau 2012). The cumulative time signatures we develop enable us to rigorously

173 assess this observation through a statistical analysis of the spatial correlations of the LFE

174 occurrence patterns. To this end, we use a hierarchical clustering algorithm (Kaufmen

175 and Rousseeuw 2005) to sort the sources into clusters based on the similarity of their

176 cumulative time signatures (equation 1). Sources within a given cluster therefore have

177 occurrence patterns that are quantitatively similar in form. We iterate the clustering

178 algorithm until we obtain clusters whose individual sources have a similarity $S$ of at least

1790.8 with the mean cumulative time signature for the cluster. A range of similarity

180 thresholds were tested, and 0.8 was chosen based upon visual inspection of the similarity

181 of the individual $C T S(t)$ within each cluster. The clustering results are not highly sensitive

182 to the choice of threshold. Note that with this threshold, some sources comprise their own

183 distinct cluster: they do not have a $\operatorname{CTS}(t)$ that is similar enough to the $\operatorname{CTS}(t)$ of any

184 other source to be joined in a cluster with another source. 
186 (north). The clusters of LFEs with synchronous occurrence patterns tend to be grouped in 187 space, suggesting the identification of coherent structures or localized asperities on the 188 deep fault interface that share the same elastic response to tectonic forcing or transient 189 pulses of slow slip. Furthermore, the dominant orientation of the clusters changes as one 190 moves north to south. The clusters to the north of Parkfield tend to be vertical structures, 191 while the clusters to the south tend to be more extensive in the horizontal direction.

192 Cluster 1S, for example, extends more than $40 \mathrm{~km}$ along strike.

193 The clusters themselves are not entirely independent. A measure of the coupling

194 between clusters can be found by computing the mean, or template cumulative time

195 signature for each cluster (Figure 2c). By calculating the correlation between the template 196 cumulative time signatures of different clusters we obtain a matrix of cluster correlation 197 coefficients (Figure S3 in the supplementary material) that provides a quantitative 198 measure of the relative strength of coupling between distinct clusters.

199 Our results are consistent with and complementary to the recent study of Shelly

200 (2015), who used a cross-correlation analysis of the source event record to investigate the 201 tendency for LFE activity to migrate from source to source at velocities of order $50 \mathrm{~km} /$ 202 hour. As one might expect, the sources within each LFE cluster we identify tend to have 203 strong migration connections as measured by Shelly (2015). The key difference between 204 our study and that of Shelly (2015) is the timescale relevant to each analysis. By

205 comparing LFE occurrence patterns at timescales longer than the timescale of order 206 minutes in Shelly (2015), we lose the temporal resolution necessary to study pairwise 207 migration patterns, but improve our ability to identify clusters of coupled LFE sources. 
209 of segmentation of tremor sources in subduction zones (Brudzinski and Allen 2007;

210 Obara 2010). This suggests that there may be certain universal features, like the spatial

211 clustering of sources on coherent elastic structures and the tendency for LFE and tremor

212 activity exhibit spatial migration (Rogers and Dragert 2003; Obara et al. 2004), that

213 typify deep crustal deformation and slow slip in all tectonic settings.

\section{$214 \quad 2.3$ Continuous and episodic styles of LFE occurrence}

215 The diversity in the forms of the cumulative time signatures makes it apparent

216 that different LFE sources can have markedly different styles of occurrence pattern. As

217 noted by Shelly (2010a) the event rate of some LFE sources is fairly consistent over time

218 (e.g., Figure 3a, bottom), while for others, LFE event rates are highly episodic, with

219 burst-like sequences of LFE events separated by long quiescent periods (e.g., Figure 3a,

220 top). To quantify the style of LFE occurrence for individual sources, we compute the

221 coefficient of variation in the daily event rate $r$ :

222

$\operatorname{cov} V_{r}=\frac{\operatorname{std}(r)}{\operatorname{mean}(r)}=\frac{\sqrt{E\left[r^{2}\right]-E[r]^{2}}}{E\left[r^{\prime}\right]}$

223 Since $\mathrm{COV}_{r}$ is simply the standard deviation in $r$ divided by the mean $r$ (for each source),

224 LFE sources with continuous occurrence styles have low $C O V_{r}$, while those with episodic

225 occurrence styles have high $\operatorname{COV}_{r}$ (Figure $\left.3 b\right)$.

226 The LFE sources appear to evolve from episodic (high $C O V_{r}$ ) to continuous (low

$227 C O V_{r}$ ) with depth (Figure 3). This finding is consistent with the observations of Shelly

228 and Johnson (2011), who use an analogous statistical metric (termed MFD75) to quantify

229 the occurrence style of LFE sources. The primary advantage of our $C O V_{r}$ metric is its

230 generality: it can be applied to compare episodicity of repeating signals in a variety of 
231 contexts, independent of the background event rate. We demonstrate such an application

232 to laboratory shear experiments in Section 3.

233 Using the $\mathrm{COV}_{r}$ metric, we find that nearby LFE sources tend to have similar

234 styles of LFE occurrence (Figure 3). There are, however, marked differences between the

235 sources to the north and south of Parkfield. Namely, the southern LFE sources are

236 bimodal - either highly episodic or highly continuous - while the northern LFE sources

237 exhibit a broader spectrum of occurrence styles between these two limiting cases.

238 Furthermore, while most clusters contain LFE sources with predominantly the same style

239 of occurrence (e.g., clusters $4 \mathrm{~N}$ and $1 \mathrm{~S}$ ), several of the larger clusters (e.g., 2S) contain

240 sources with diverse occurrence styles (Figure S3 in the supplementary material).

\section{3. Discussion: Recurrence Spectra of LFE Sources and Comparison to}

\section{Laboratory Shear Experiments}

243 These systematic differences in the styles of LFE occurrence are intriguing, and

244 motivate a closer examination of the fine features of the occurrence patterns of individual

245 sources. For a source that has successive LFE events at times $t_{m}$ and $t_{m+1}$, we can define

246 the recurrence rate at the mean event time, $\left(t_{m}+t_{m+1}\right) / 2$, to be the reciprocal of the

247 recurrence interval between the two event times: $1 /\left(t_{m+1}-t_{m}\right)$. In Figure 4 , we show the

248 full spectrum of recurrence rates for two representative LFE sources with contrasting

249 occurrence styles: episodic and continuous (these same sources are featured in Figure 3).

250 The occurrence style of an individual source can be easily distinguished by

251 examining its spectra of recurrence rates. The bursts of LFE activity associated with

252 episodic sources (high $C O V_{r}$ ) have recurrence rate spectra that are self-similar in nature,

253 with rates that span time-scales over 6 orders of magnitude. In contrast, the sources with 
254 continuous occurrence styles (low $C O V_{r}$ ) tend to have bimodal recurrence spectra and

255 notably shorter maximum recurrence intervals.

256 We can gain insight into the physical processes underlying these differences in

257 recurrence rate spectra through comparison to laboratory studies of shear slip in granular

258 media under a variety of experimental conditions (Johnson et al. 2012; Johnson et al.

259 2013, see Appendix for further experimental details). In such studies, there is a clear

260 transition from episodic to continuous occurrence of acoustic emission (slip events that

261 we posit to be analogous to LFEs) with decreasing normal stress (Figure 4, panels (e) and

262 (f)). This transition corresponds to an evolution from an episodic, or stick-slip-like, slip

263 regime to a continuous, or stable-sliding slip regime (Marone 1998).

264 We can quantify this observation more precisely by computing the coefficient of

265 variation in the event rate of acoustic emission for laboratory experiments with different

266 levels of applied normal stress (Figure 5). $C O V_{r}$ for the laboratory data is computed in a

267 similar fashion as for the LFE data set (equation 2) except the rate of acoustic emission is

268 computed in time bins of $0.25 \mathrm{~s}$, instead of the daily time bins used for the LFE data set.

269 The results are not highly sensitive to the choice of bin size - we use bins of $0.25 \mathrm{~s}$ for the

270 laboratory data to ensure that we have approximately the same number of time bins for

271 the computation of lab $C O V_{r}$ as we have for the computation of LFE $C O V_{r}$.

272 The rheology and physical conditions of the deep SAF are undoubtedly more

273 complex than that of the laboratory experiments. It is, however, plausible that many of

274 the same physical processes that control the occurrence styles of acoustic emission in

275 laboratory shear experiments (e.g., normal stress, shearing rate, and fault gouge

276 composition) may be responsible for the distinctive features in the patterns of LFE 
277 occurrence. For example, LFE source regions where local fault conditions include lower

278 effective stress (e.g., higher pore pressure) or more fine-grained (e.g., clay-like) fault

279 gouge material would tend to exhibit continuous styles of LFE occurrence (i.e., a stable

280 sliding regime). Source regions with higher effective stress or coarser gouge material

281 would tend to exhibit episodic LFE occurrence (i.e., a stick-slip-like regime).

282 Laboratory shear experiments on granular, gouge-like materials also provide an

283 intriguing analog to the natural occurrence of LFEs because of the importance of slip-

284 induced dilatancy to the frictional dynamics in both contexts. In laboratory experiments,

285 the fault gouge tends to dilate with increasing shear rate (Samuelson et al., 2009). Slip-

286 induced dilatancy has likewise been hypothesized to be an important mechanism in

287 regulating the frictional stability of slow slip and tremor (and hence, LFEs) within the

288 earth (Segall et al. 2010; Shelly 2015), as dilation reduces the pore pressure (and

289 therefore, the effective stress) on the fault interface.

290 We further note that the episodic LFE sources tend to be shallower than those

291 with continuous occurrence styles (Figures 3 and 5), suggesting an evolution from brittle,

292 unstable slip behavior to ductile, stable slip behavior with depth. Similar observations

293 have been documented in tremor zones of Japan (Obara et al. 2010) and Cascadia (Wech

294 and Creager 2011), and have broader implications for seismic hazard in the Parkfield

295 region. The upper-crustal section of the SAF to the south of Parkfield is currently locked,

296 having last ruptured during the $1857 \mathrm{M}_{\mathrm{w}}$ 7.9 Fort Tejon earthquake (Sieh 1978). This

297 locked section is situated directly above a cluster of LFE sources with episodic but quite

298 frequent occurrence (Figure 3). Slow slip transients in the deep fault system, through 
299 elastic coupling to the adjacent upper crust, may therefore load this locked section and

300 play an important role in the nucleation of a future large earthquake (Shelly et al. 2007).

\section{4. Conclusions}

$302 \quad$ No geodetic signals from deep slow slip events have been directly observed along

303 the Parkfield portion of the SAF (Johnston et al. 2006), though episodes of LFEs and

304 tremor have been used to infer deep slow slip events reminiscent of ETS in Cascadia and

305 Japan (Guilhem and Nadeau, 2012). Consequently, LFE sources and their associated

306 occurrence patterns are important tools for probing the stress, frictional, and slip

307 characteristics of the deep SAF. We have conducted a detailed statistical analysis of the

308 patterns of LFE occurrence for 88 individual sources near Parkfield. Our analysis

309 suggests that the regions to the north and south of Parkfield are decoupled. Each of these

310 regions is assembled from discrete elastic structures or asperities in which clusters of

311 LFE sources with similar occurrence patterns reside. These sources have distinctive, fine

312 features in their occurrence patterns that can be characterized by their spectra of event

313 occurrence rates. Guided by laboratory experiments, we posit that these fine features

314 reflect the slip and stress conditions at the LFE source locations. The resulting map of

315 slip and stress conditions revealed through our multiscale analysis of LFE occurrence

316 patterns provides a basis for assessing deformational processes in the deep SAF.

318 Appendix: Description of laboratory acoustic emission experiments

319 The acoustic emission (AE) recurrence data used for this study comes from

320 double-direct shear experiments (Marone 1998) using a servo-hydraulic testing machine

321 (Johnson et al. 2012; Johnson et al. 2013). Our analysis of AE recurrence rates is based 
322 on experiment p2394 (Johnson et al. 2013), in which two layers of simulated fault gouge

323 were subjected to a shear stress using a double-direct shearing apparatus. The simulated

324 fault gouge consisted of class IV spheres (with dimensions from $105-149 \mu \mathrm{m}$ ), with

325 initial layer thicknesses of $2 \times 4 \mathrm{~mm}$ (two layers). The drive block vertical displacement

326 rate was $5 \mu \mathrm{m} / \mathrm{s}$, corresponding to a strain rate of approximately $1.2 \times 10^{-3} / \mathrm{s}$. The

327 applied normal stress was incrementally increased from $2 \mathrm{MPa}$ to $8 \mathrm{MPa}$ and then

328 decreased back to $2 \mathrm{MPa}$, in increments of $1 \mathrm{MPa}$. The time series of AE recurrence rate

329 shown in Figure 4e and Figure 4f are from 3-minute intervals from the downgoing stress

330 steps in experiment p2394, with normal loads of $5 \mathrm{MPa}$ and $3 \mathrm{MPa}$, respectively.

331 The shearing apparatus in experiment p2394 was servocontrolled so that constant

332 normal stress and displacement rate of the drive block were maintained at $\pm 0.1 \mathrm{kN}$ and

$333 \pm 0.1 \mu \mathrm{m} / \mathrm{s}$, respectively. The shearing stresses were then measured with a strain gauge,

334 and the loads, displacements and stresses were monitored by computer at $10 \mathrm{kHz}$

335 sampling frequency. We also used another acquisition system to record the acoustic

336 acceleration, shear stress and layer thickness at a sampling frequency of $330 \mathrm{kHz}$.

337 Detailed experiment parameters can be found in (Johnson et al. 2013).

\section{Acknowledgments}

340 The LFE data used for this work was derived from the updated LFE catalog

341 maintained by David Shelly (Shelly and Hardebeck 2010). Data from laboratory slip

342 experiments was obtained and used with permission from Paul Johnson (Johnson et al.

343 2013). Both data sets may be accessed by contacting the corresponding author directly. 
345 Laboratory, and is based upon work supported by the National Science Foundation

346 Graduate Research Fellowship Program (NSFGRFP) under grant number DGE-1144086.

347 Discussions with W. Frank, A. Delorey, J. Gomberg, and D. Shelly helped to guide this

348 study.. We are also grateful for the thoughtful reviews provided by A. Thomas, an

349 anonymous reviewer, and the Editor, all of which greatly improved the manuscript. 350

\section{$351 \quad$ References}

352 Bennington, N., C. Thurber, K. Feigl, and J. Murray-Moraleda (2011). Aftershock Distribution as 353 a Constraint on the Geodetic Model of Coseismic Slip for the 2004 Parkfield Earthquake, 354 Pure Appl. Geophys. 168, no. 10, 1553-1565, doi: 10.1007/s00024-010-0214-x.

355 Bostock, M. G., A. A. Royer, E. H. Hearn, and S. M. Peacock (2012). Low frequency

356 earthquakes below southern Vancouver Island, Geochem. Geophys.Geosyst. 13, no. 11, 357 Q11007, doi: 10.1029/2012GC004391.

358 Brudzinski, M. R., and R. M. Allen (2007). Segmentation in episodic tremor and slip all along 359 Cascadia, Geology 35, no. 10, 907-910, doi: 10.1130/G23740A.1.

360 Frank, W. B., N. M. Shapiro, V. Kostoglodov, A. L. Husker, M. Campillo, J. S. Payero, and G. A. 361 Prieto (2013). Low-frequency earthquakes in the Mexican Sweet Spot, Geophys. Res. Lett. 36240 , no. 11, 2661-2666, doi: 10.1002/grl.50561.

363 Guilhem, A., and R. M. Nadeau (2012). Episodic tremors and deep slow-slip events in Central 364 California, Earth Planet. Sci. Lett. 357-358, no. 0, 1-10, doi: 10.1016/j.eps1.2012.09.028. 
365 Johnson, P., B. Carpenter, M. Knuth, B. Kaproth, P. Le Bas, E. Daub, and C. Marone (2012).

366 Nonlinear dynamical triggering of slow slip on simulated earthquake faults with

367 implications to Earth, J. Geophys. Res. 117, B04310, doi: 10.1029/2011JB008594.

368 Johnson, P. A., B. Ferdowsi, B. M. Kaproth, M. Scuderi, M. Griffa, J. Carmeliet, R. A. Guyer, P.

369 Le Bas, D. T. Trugman, and C. Marone (2013). Acoustic emission and microslip precursors

370 to stick-slip failure in sheared granular material, Geophys. Res. Lett. 40, no. 21,

371 2013GL057848, doi: 10.1002/2013GL057848.

372 Johnston, M. J. S., R. D. Borcherdt, A. T. Linde, and M. T. Gladwin (2006). Continuous

373 Borehole Strain and Pore Pressure in the Near Field of the 28 September 2004 M 6.0

374 Parkfield, California, Earthquake: Implications for Nucleation, Fault Response, Earthquake

375 Prediction, and Tremor, Bull. Seismol. Soc. Am. 96, no. 4B, S56-S72, doi:

$376 \quad 10.1785 / 0120050822$.

377 Kaufmen, L., and P. Rousseeuw (2005). Finding Groups in Data: An Introduction to Cluster

378 Analysis, Wiley-Interscience, Indianapolis, IN.

379 Marone, C. (1998). Laboratory-derived friction laws and their application to seismic faulting, 380 Annu. Rev. Earth Planet. Sci. 26, no. 1, 643-696.

381 Nadeau, R. M., and D. Dolenc (2005). Nonvolcanic Tremors Deep Beneath the San Andreas

$382 \quad$ Fault, Science 307, no. 5708, 389-389, doi: 10.1126/science.1107142.

383 Nadeau, R. M., and A. Guilhem (2009). Nonvolcanic tremor evolution and the San Simeon and

384 Parkfield, California, earthquakes, Science 325, no. 5937, 191-193, doi:

$385 \quad 10.1126 /$ science.1174155. 
386 Obara, K. (2010). Phenomenology of deep slow earthquake family in southwest Japan:

387 Spatiotemporal characteristics and segmentation, Journal of Geophysical Research: Solid

388 Earth 115, no. B8, B00A25, doi: 10.1029/2008JB006048.

389 Obara, K., H. Hirose, F. Yamamizu, and K. Kasahara (2004). Episodic slow slip events

390 accompanied by non-volcanic tremors in southwest Japan subduction zone, Geophys. Res.

391 Lett. 31, no. 23, L23602, doi: 10.1029/2004GL020848.

392 Obara, K., S. Tanaka, T. Maeda, and T. Matsuzawa (2010). Depth-dependent activity of non393 volcanic tremor in southwest Japan, Geophys.Res.Lett. 37, no. 13, L13306, doi:

$394 \quad 10.1029 / 2010$ GL043679.

395 Rivet, D., M. Campillo, N. M. Shapiro, V. Cruz-Atienza, M. Radiguet, N. Cotte, and V.

396 Kostoglodov (2011). Seismic evidence of nonlinear crustal deformation during a large slow

397 slip event in Mexico, Geophys. Res. Lett. 38, no. 8, L08308, doi: 10.1029/2011GL047151.

398 Rogers, G., and H. Dragert (2003). Episodic tremor and slip on the Cascadia subduction zone:

399 The chatter of silent slip, Science 300, no. 5627, 1942-1943, doi: 10.1126/science.1084783.

400 Rubinstein, J. L., D. R. Shelly, and W. L. Ellsworth (2010). Non-volcanic tremor: A window into 401 the roots of fault zones, in New Frontiers in Integrated Solid Earth Sciences, S. Cloetingh, 402 and J. Negendank (Editors), Springer, Amsterdam, Netherlands. 287-314.

403 Samuelson, J., D. Elsworth, and C. Marone (2009). Shear-induced dilatancy of fluid-saturated 404 faults: Experiment and theory, Journal of Geophysical Research: Solid Earth 114, no. B12, 405 B12404, doi: 10.1029/2008JB006273.

406 Scholz, C. H. (2002). The mechanics of earthquakes and faulting, Cambridge University Press, 407 Cambridge, UK. 
408 Segall, P., and A. M. Bradley (2012). Slow-slip evolves into megathrust earthquakes in 2D

409 numerical simulations, Geophys. Res. Lett. 39, no. 18, L18308, doi:

$410 \quad 10.1029 / 2012$ GL052811.

411 Segall, P., A. M. Rubin, A. M. Bradley, and J. R. Rice (2010). Dilatant strengthening as a

412 mechanism for slow slip events, J. Geophys. Res. 115, no. B12, - B12305, doi:

$413 \quad 10.1029 / 2010 J B 007449$.

414 Shelly, D. R. (2010a). Periodic, chaotic, and doubled earthquake recurrence intervals on the deep 415 San Andreas Fault, Science 328, no. 5984, 1385-1388, doi: 10.1126/science.1189741.

416 Shelly, D. R., G. C. Beroza, and S. Ide (2007). Non-volcanic tremor and low-frequency

417 earthquake swarms, Nature 446, no. 7133, 305-307, doi: 10.1038/nature05666.

418 Shelly, D. R., G. C. Beroza, S. Ide, and S. Nakamula (2006). Low-frequency earthquakes in

419 Shikoku, Japan, and their relationship to episodic tremor and slip, Nature 442, no. 7099, $420 \quad$ 188-191, doi: 10.1038/nature04931.

421 Shelly, D. R., Z. Peng, D. P. Hill, and C. Aiken (2011). Triggered creep as a possible mechanism 422 for delayed dynamic triggering of tremor and earthquakes, Nat. Geosci. 4, no. 6, 384-388, 423 doi: $10.1038 /$ NGEO1141.

424 Shelly, D. R. (2015). Complexity of the deep San Andreas Fault zone defined by cascading 425 tremor, Nat. Geosci. 8, no. 2, 145-151, doi: 10.1038/ngeo2335.

426 Shelly, D. R. (2010b). Migrating tremors illuminate complex deformation beneath the 427 seismogenic San Andreas fault, Nature 463, no. 7281, 648-652, doi: 10.1038/nature08755. 
428 Shelly, D. R. (2009). Possible deep fault slip preceding the 2004 Parkfield earthquake, inferred 429 from detailed observations of tectonic tremor, Geophys. Res. Lett. 36, no. 17, L17318, doi: $430 \quad 10.1029 / 2009$ GL039589.

431 Shelly, D. R., W. L. Ellsworth, T. Ryberg, C. Haberland, G. S. Fuis, J. Murphy, R. M. Nadeau, 432 and R. Bürgmann (2009). Precise location of San Andreas Fault tremors near Cholame, 433 California using seismometer clusters: Slip on the deep extension of the fault? Geophys. Res. $434 \quad$ Lett. 36, no. 1, L01303, doi: 10.1029/2008GL036367.

435 Shelly, D. R., and J. L. Hardebeck (2010). Precise tremor source locations and amplitude 436 variations along the lower-crustal central San Andreas Fault, Geophys. Res. Lett. 37, no. 14, $437 \quad$ L14301, doi: 10.1029/2010GL043672.

438 Shelly, D. R., and K. M. Johnson (2011). Tremor reveals stress shadowing, deep postseismic 439 creep, and depth-dependent slip recurrence on the lower-crustal San Andreas fault near $440 \quad$ Parkfield, Geophys. Res. Lett. 38, no. 13, L13312, doi: 10.1029/2011GL047863.

441 Sieh, K. E. (1978). Central California foreshocks of the great 1857 earthquake, Bull. Seismol. Soc. Am. 68, 1731-1749.

443 Thomas, A. M., R. Bürgmann, D. R. Shelly, N. M. Beeler, and M. L. Rudolph (2012). Tidal 444 triggering of low frequency earthquakes near Parkfield, California: Implications for fault 445 mechanics within the brittle-ductile transition, J. Geophys. Res. 117, no. B5, B05301, doi: $446 \quad 10.1029 / 2011 J B 009036$.

447 Tse, S. T., and J. R. Rice (1986). Crustal earthquake instability in relation to the depth variation 448 of frictional slip properties, Journal of Geophysical Research: Solid Earth 91, no. B9, 9452449 9472, doi: 10.1029/JB091iB09p09452. 
450 Wech, A. G., and K. C. Creager (2011). A continuum of stress, strength and slip in the Cascadia 451 subduction zone, Nat. Geosci. 4, no. 9, 624-628, doi: 10.1038/ngeo1215.

452 Wu, C., D. R. Shelly, J. Gomberg, Z. Peng, and P. Johnson (2013). Long-term changes of

453 earthquake inter-event times and low-frequency earthquake recurrence in central California,

454 Earth Planet. Sci. Lett. 368, no. 0, 144-150, doi: 10.1016/j.eps1.2013.03.007.

455 


\section{Figure Captions: Figures 1 - 5}

459 Figure 1: Overview of the Parkfield study region and LFE seismicity. (a) Cross-sectional

460 and (b) map view of the positions of the 88 LFE sources (red circles). The hypocentral

461 position of the $\mathrm{M}_{\mathrm{w}} 6.02004$ Parkfield earthquakes is denoted in (a) with a yellow star.

462 The locations of the HRSN and NCSN stations (yellow and green triangles, respectively),

463 and the moment tensor solution and rupture zone for the 2004 Parkfield earthquake

464 (Bennington et al. 2011), are shown in (b). (c) Space-time plot of the periods at which the

465 LFE event rate exceeds 3 standard deviations above the mean rate. Each LFE source is

466 displayed as function of along-strike source position $Z$ from Parkfield $\left(35.9^{\circ} \mathrm{N}, 120.4^{\circ} \mathrm{W}\right.$;

$467 \quad Z=0)$.

469 Figure 2: LFE cluster locations and template cumulative time signatures. (a) Positions of

470 the 48 LFE sources south of Parkfield. The LFE sources are color-coded by cluster, and

471 markers without coloration correspond to LFE sources that do not belong to a cluster. (b)

472 Similar to panel (a), but for the 40 LFE sources north of Parkfield. (c) Template

473 cumulative time signatures for all LFE clusters. The individual cumulative time

474 signatures for all sources in clusters $2 \mathrm{~S}$ and $4 \mathrm{~N}$ are shown as examples in the inset and 475 are separated from the template signature by a dotted line.

477 Figure 3: LFE occurrence styles and event rate COV. (a) Comparison of the time 478 evolution of the cumulative fraction of LFE occurrences at sources 78 and 80 (both 
479 located to the south of Parkfield). Source 78 has a highly episodic occurrence style, while

480 source 80 has a highly continuous occurrence style. (b) Comparison of the daily event

481 rate (normalized by the mean rate) for sources 78 and 80 . (c) Rate coefficient of variation

$482\left(\mathrm{COV}_{r}\right)$ for the LFE sources south of Parkfield, with source 78 and 80 labeled for

483 reference. Cooler colors correspond to higher $\mathrm{COV}_{r}$. The cross-section is exaggerated in

484 the vertical direction for clarity. (d) Similar to (c), but for the LFE sources north of

485 Parkfield.

486

487 Figure 4: Recurrence rate spectra. Logarithm of the recurrence rates for LFE sources 78

488 (a), and 80 (b), are plotted as a function of time for the time interval between 500 and

4891500 days after January $1^{\text {st }}, 2006$. Source 78 has a highly episodic occurrence style, while

490 source 80 has a highly continuous occurrence style. The corresponding probability

491 density functions (log-log scale) for the entire observation time interval are shown in (c)

492 and (d), respectively. Logarithm of the acoustic emission event recurrence rates are

493 plotted for 3-minute time intervals of laboratory shear experiment p2394, with applied

494 normal loads of (e) $5 \mathrm{MPa}$ and (f) $3 \mathrm{MPa}$. Measured shear stress (arbitrary units) is

495 plotted for reference. Note the strong correlation of acoustic emission with stress drop in 496 (e).

498 Figure 5: Acoustic emission $C O V_{r}$ and depth-dependence of LFE $C O V_{r}$ (a) Comparison 499 of the time evolution of the cumulative fraction of acoustic emissions (AE) for laboratory 500 shear experiments on granular media with $5 \mathrm{MPa}$ (top) and $3 \mathrm{MPa}$ (bottom) normal stress.

501 (b) Comparison of the AE event rate (computed every $0.25 \mathrm{~s}$, and normalized by the mean 
502 rate) for the same laboratory experiments. (c) $C O V_{r}$ as a function of depth for LFE

503 sources south (pink circles) and north (green squares) of Parkfield.

504 


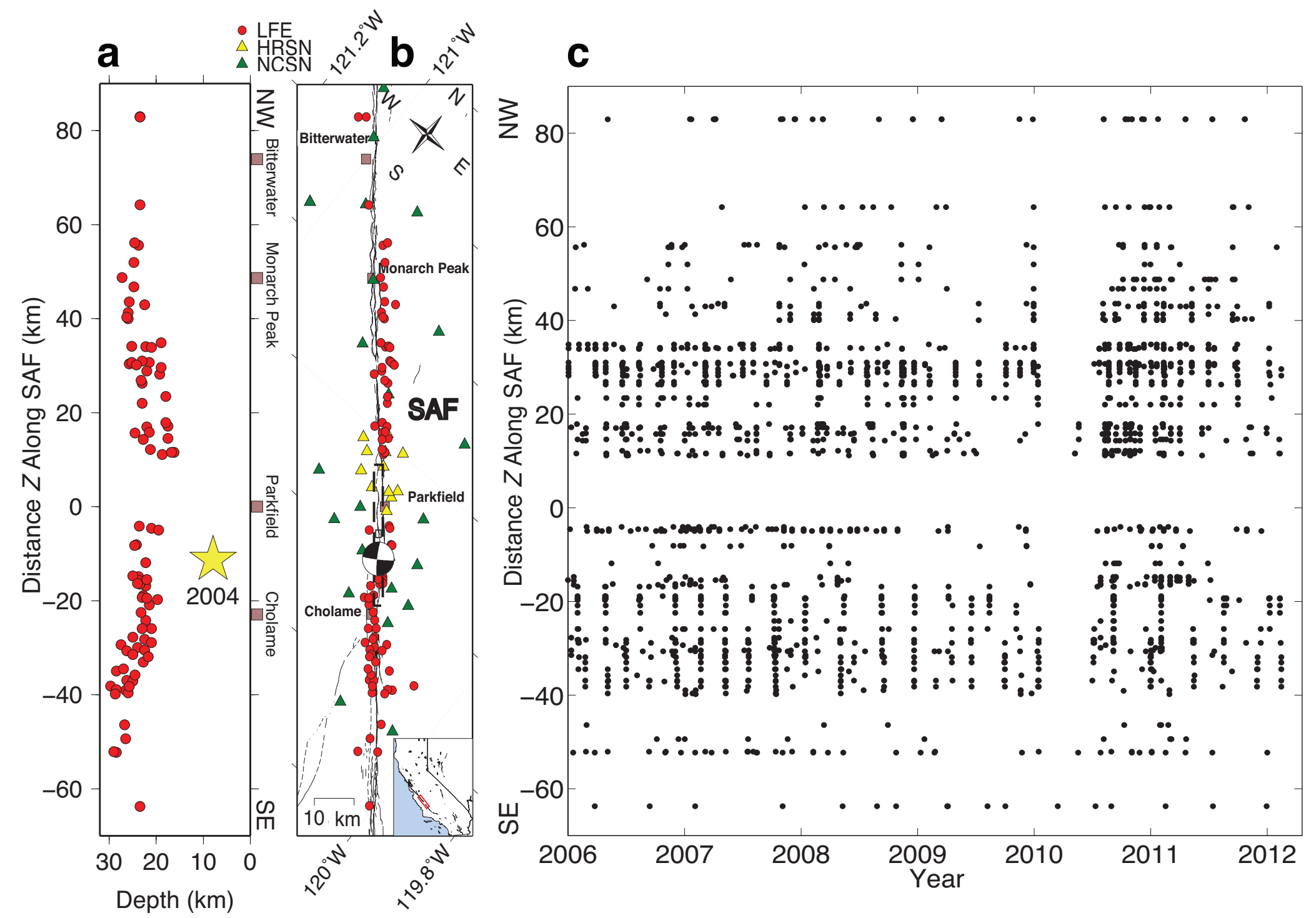


a

$\begin{array}{llllll}1 S & 2 S & 3 S & 4 S & 5 S & 6 S\end{array}$

b

$\begin{array}{llllll}1 \mathrm{~N} & 2 \mathrm{~N} & 3 \mathrm{~N} & 4 \mathrm{~N} & 5 \mathrm{~N}\end{array}$

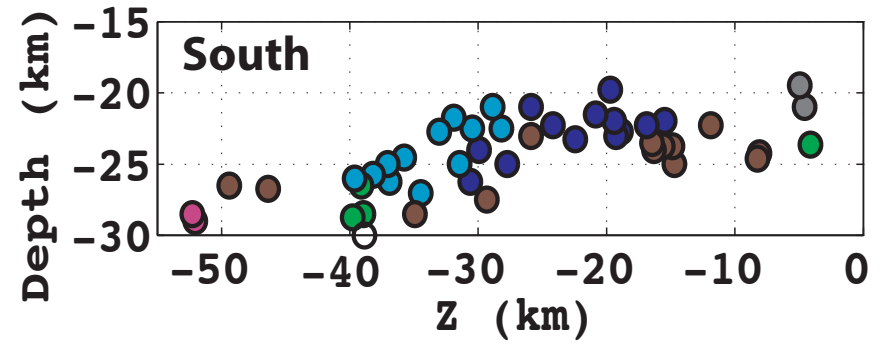

C

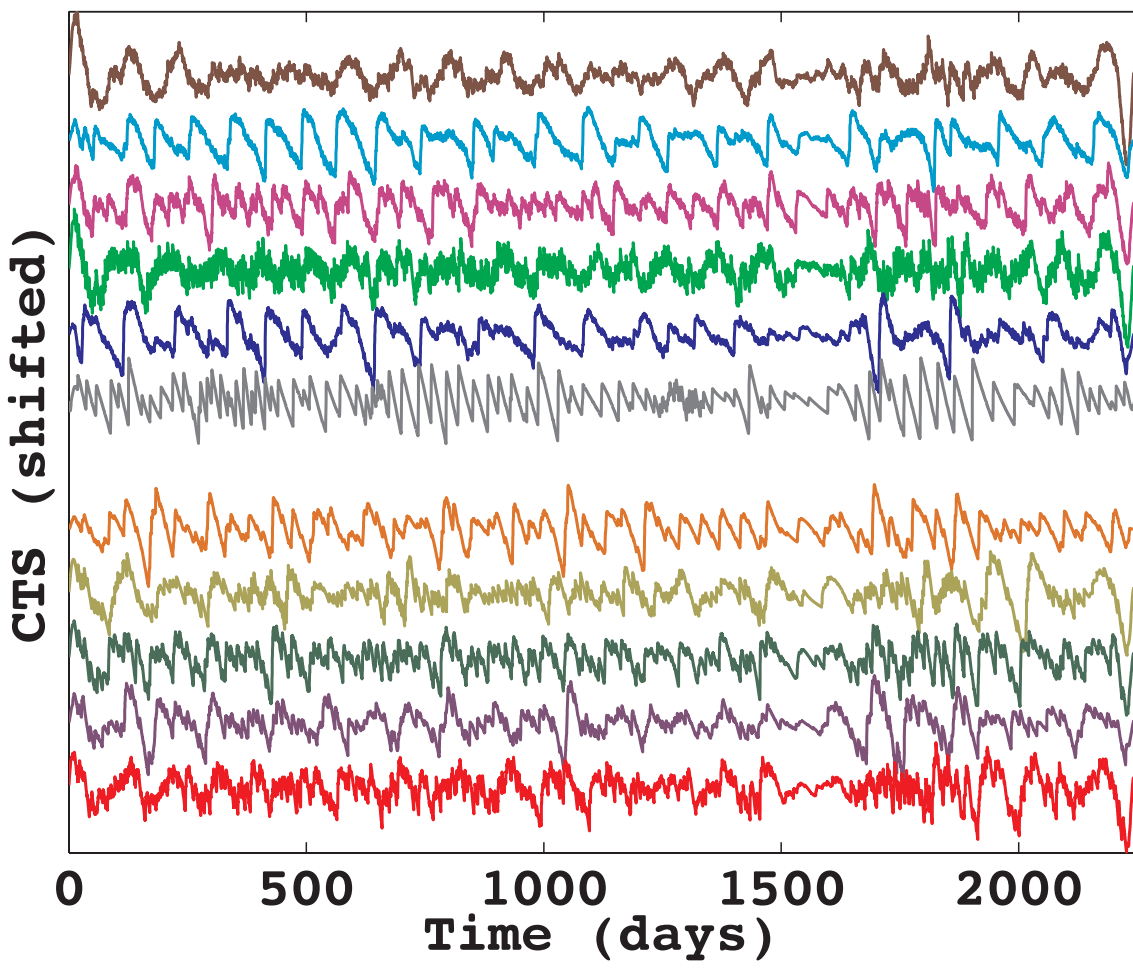

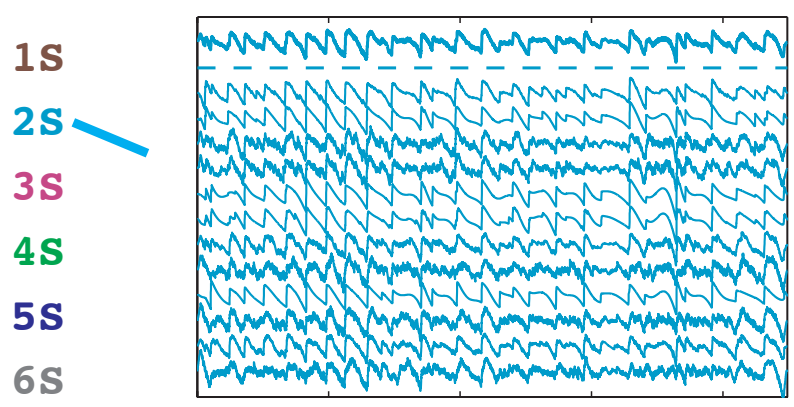

$1 N$

$2 \mathrm{~N}$

$3 \mathrm{~N}$

$4 N$

$5 \mathrm{~N}$

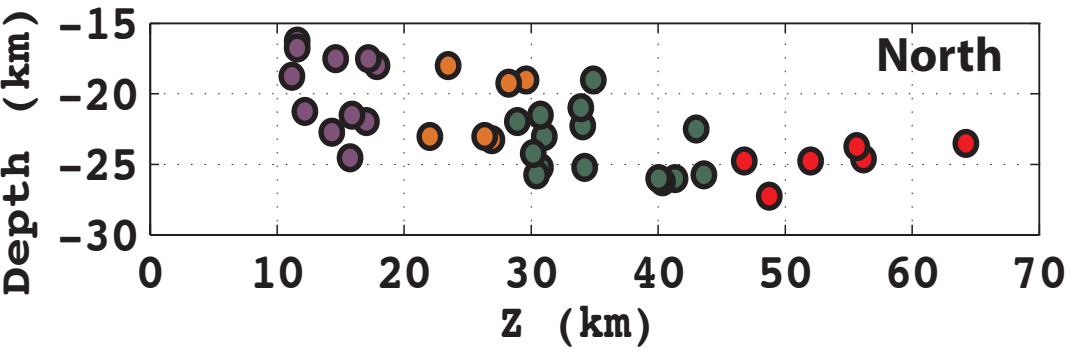


Figure 3
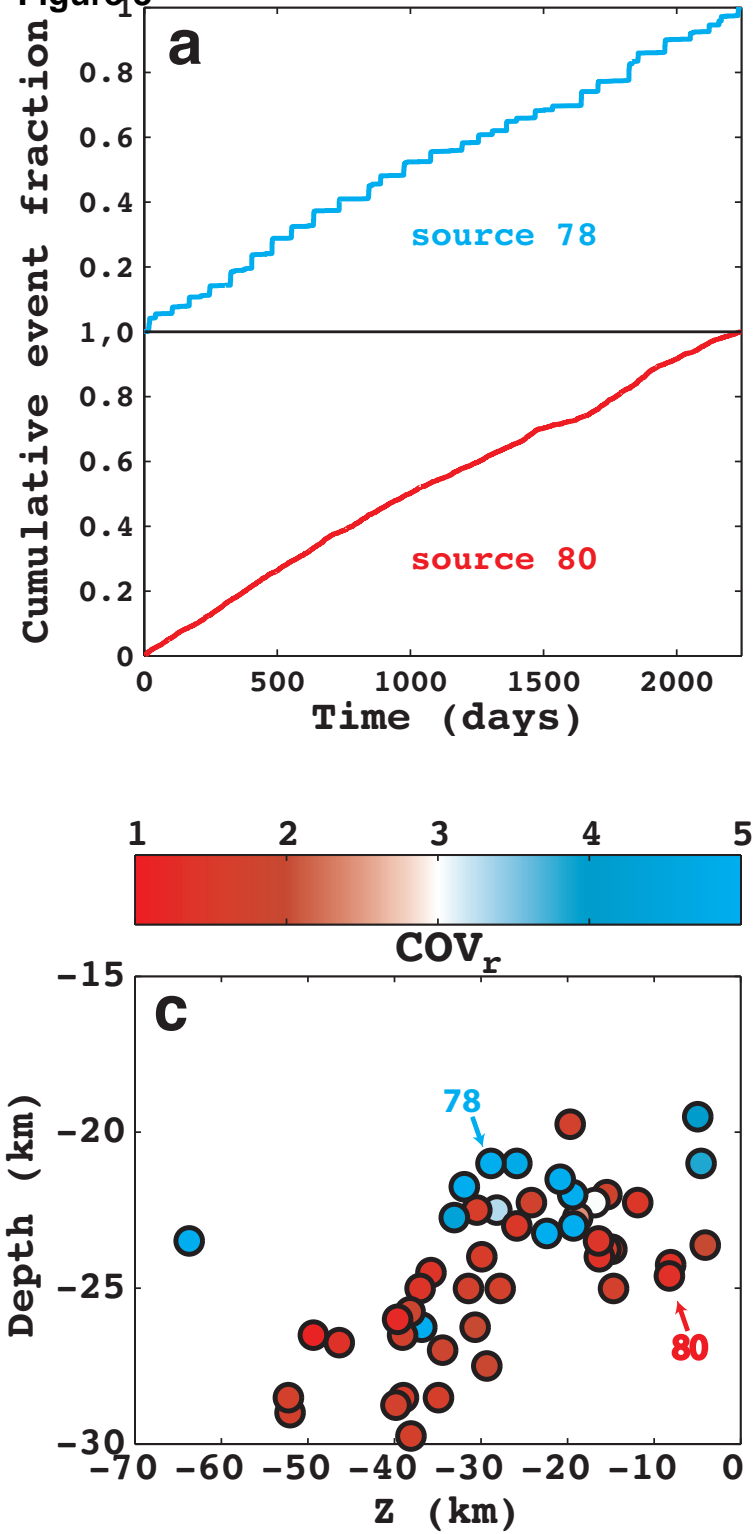

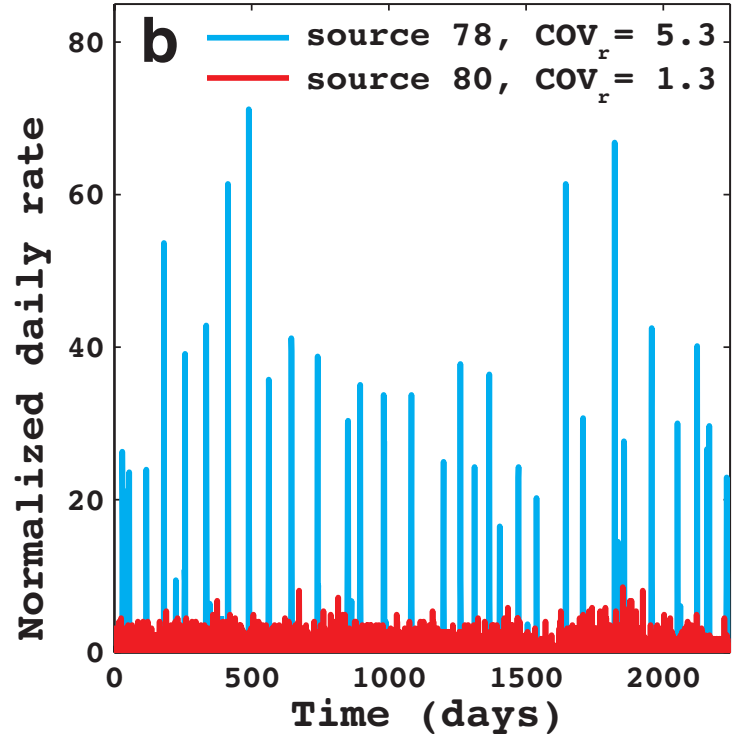

1

2

3

4

5

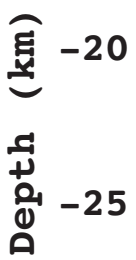

$-15 \quad \operatorname{COV}_{\mathbf{r}}$

d 8080

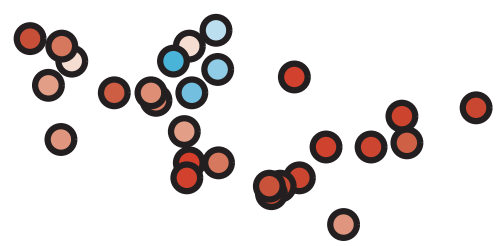

$-30$

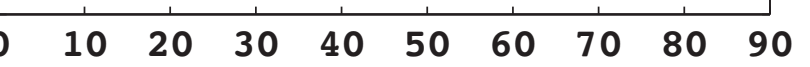

$\mathrm{Z}(\mathrm{km})$ 

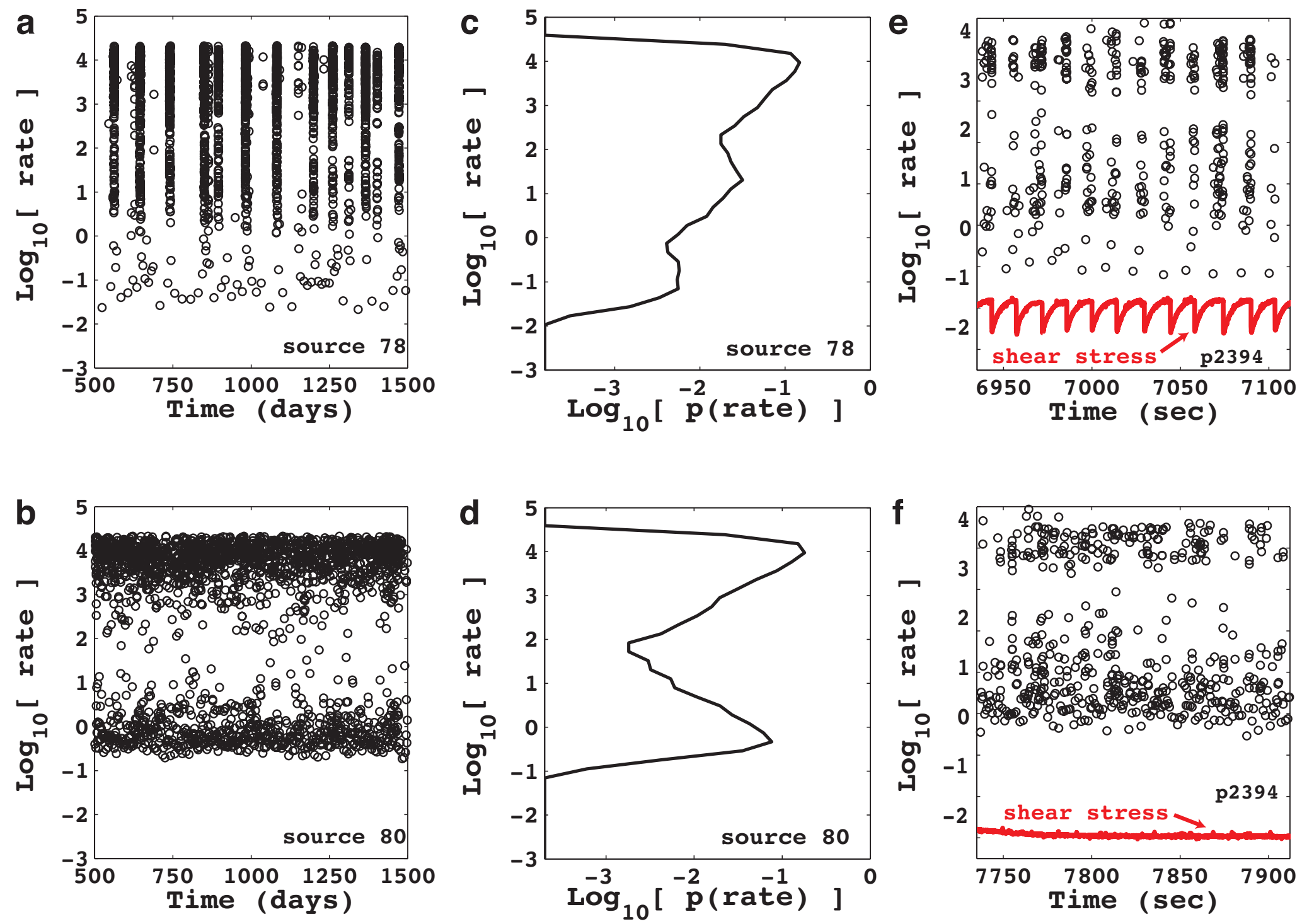


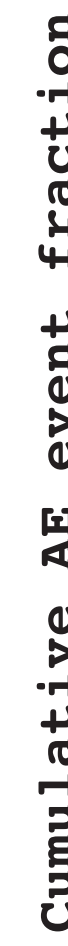

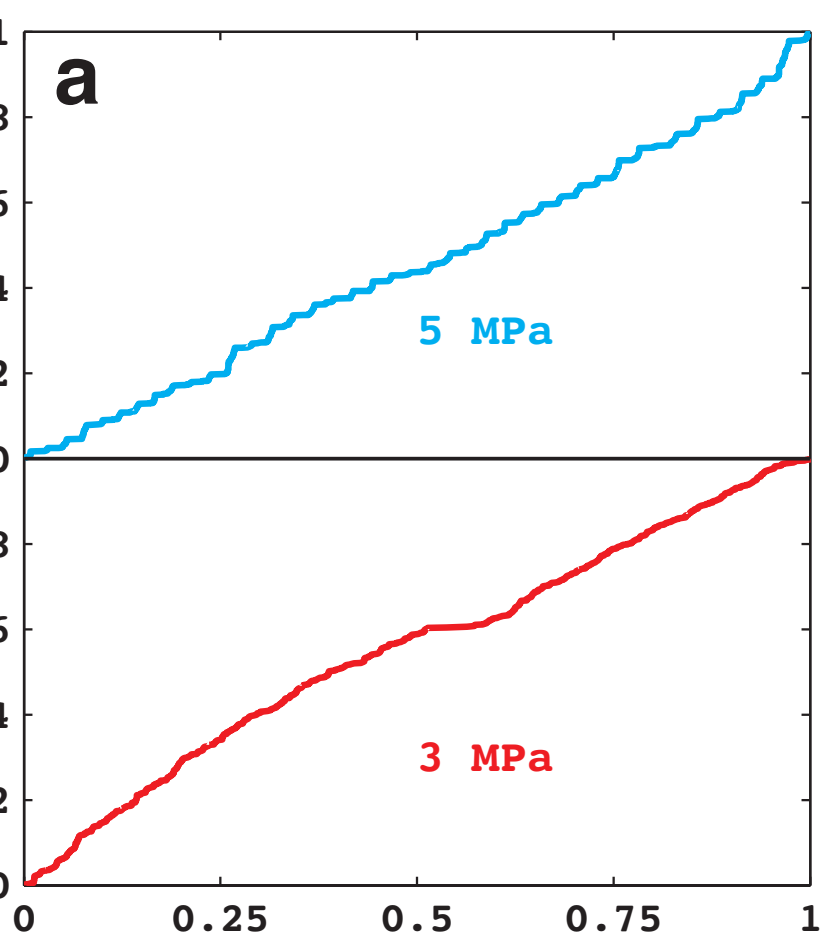

Relative experimental time

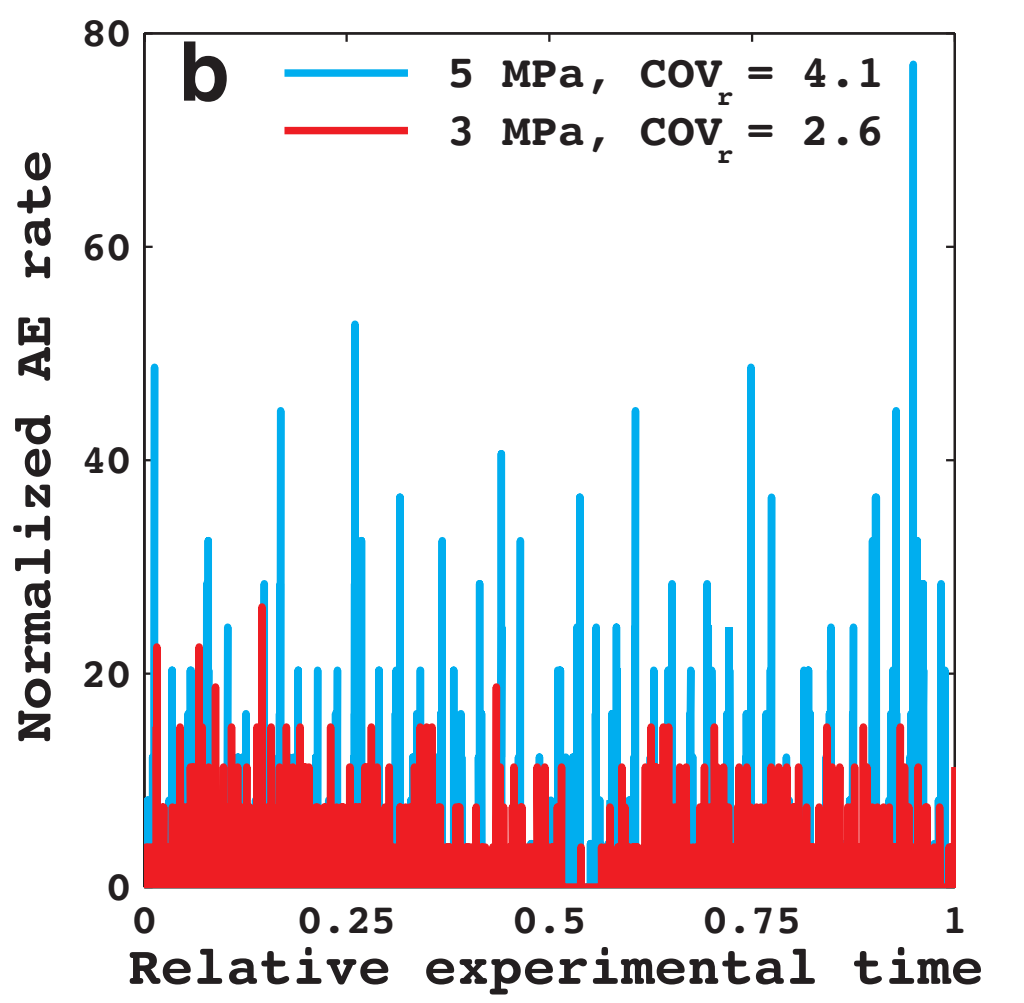

Southern sources

- Northern sources

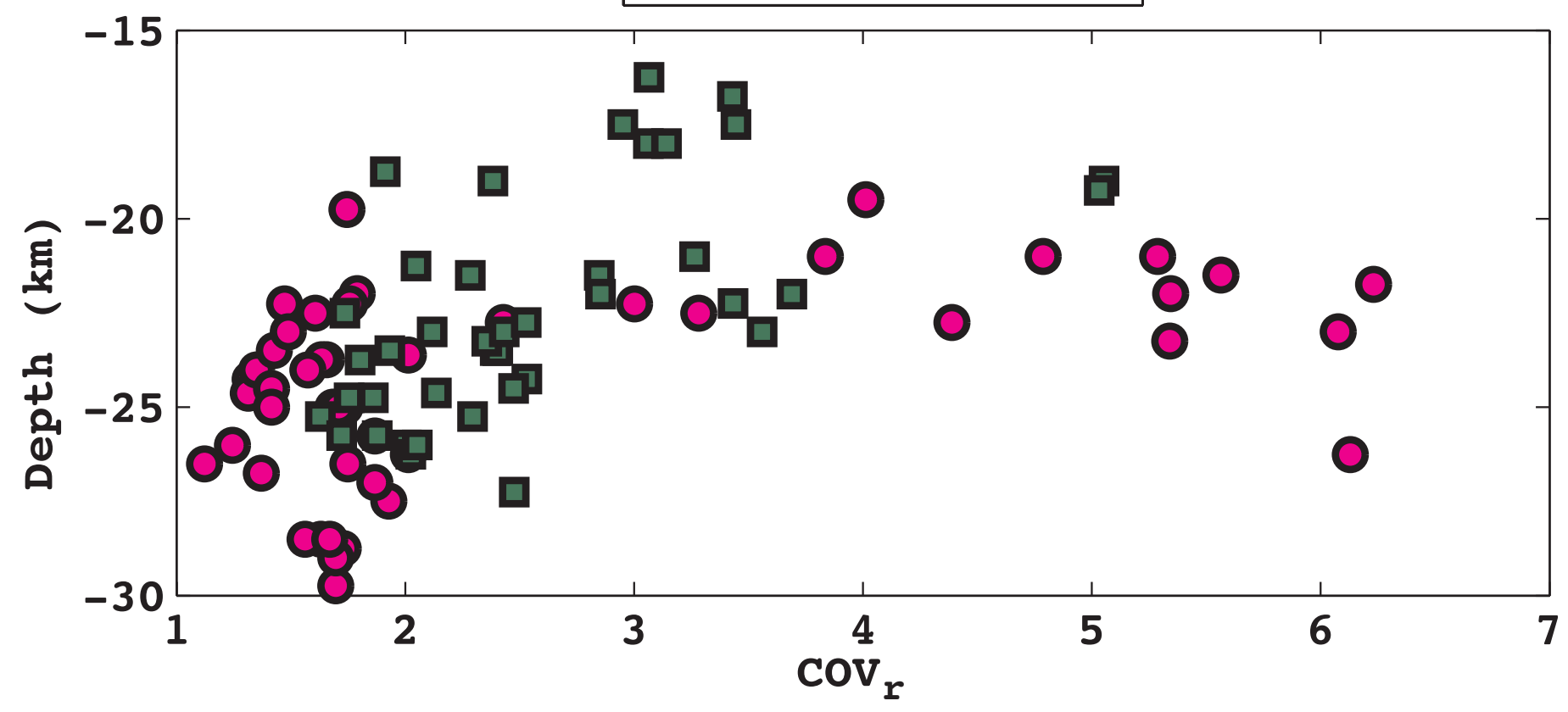

